"Supporting information"

\title{
Synthesis of Novel Caspase Inhibitors for Characterization of the Active Caspase Proteome in Vitro and in Vivo
}

\author{
Alexander J. Henzing, ${ }^{\text {a }}$ Helen Dodson ${ }^{\text {a }}$, Joel M. Reid, ${ }^{\mathrm{b}}$ Scott H. Kaufmann, ${ }^{\mathrm{b}, \mathrm{c}}$ Robert L. \\ Baxter $^{\mathrm{d}}$ and William C. Earnshaw ${ }^{\mathrm{a}, e, \mathrm{f}}$
}

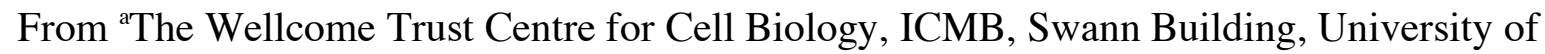
Edinburgh, Mayfield Road, Edinburgh, EH9 3JR, United Kingdom, Departments of ${ }^{\mathrm{b}}$ Oncology and ${ }^{\mathrm{c} M o l e c u l a r}$ Pharmacology, Mayo Clinic College of Medicine, Rochester, MN 55905, and ${ }^{\mathrm{d} S c h o o l ~ o f ~ C h e m i s t r y, ~ J o s e p h ~ B l a c k ~ B u i l d i n g, ~ U n i v e r s i t y ~ o f ~ E d i n b u r g h, ~ W e s t ~ M a i n s ~ R o a d, ~}$ Edinburgh EH9 3JJ, United Kingdom 


\section{Supplemental Data}

\section{$\underline{\text { HPLC of 12a-12d and } 13}$}

Waters C-18 column $(10 \mu, 150$ x $1 \mathrm{~mm})$ eluted isocratically with $\mathrm{AcCN}: \mathrm{H}_{2} \mathrm{O}: \mathrm{HCOOH}$ (2:98:0.1, $1 \mathrm{ml} / \mathrm{min})$ for $2 \mathrm{~min}$ and then with a gradient to $60 \% \mathrm{AcCN}: \mathrm{H}_{2} \mathrm{O}: \mathrm{HCOOH}(97: 3: 0.05)$ over $20 \mathrm{~min}$. Detection was at $214 \mathrm{~nm}$.

\section{2a}

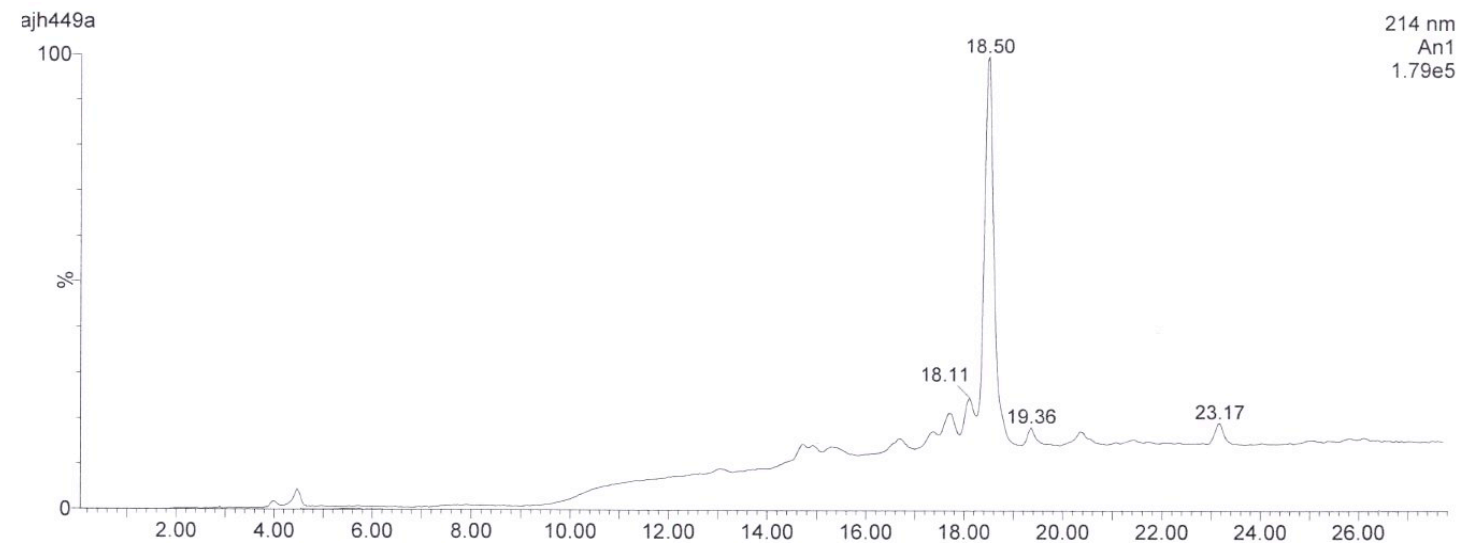

\section{2b}

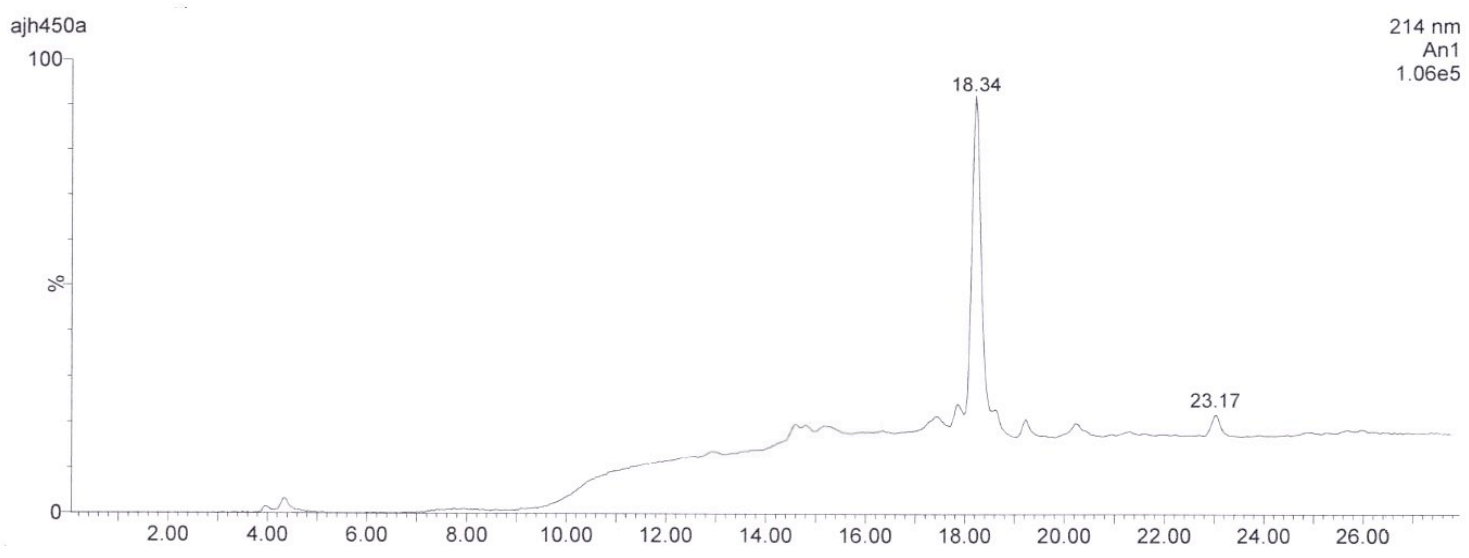




\section{2c}

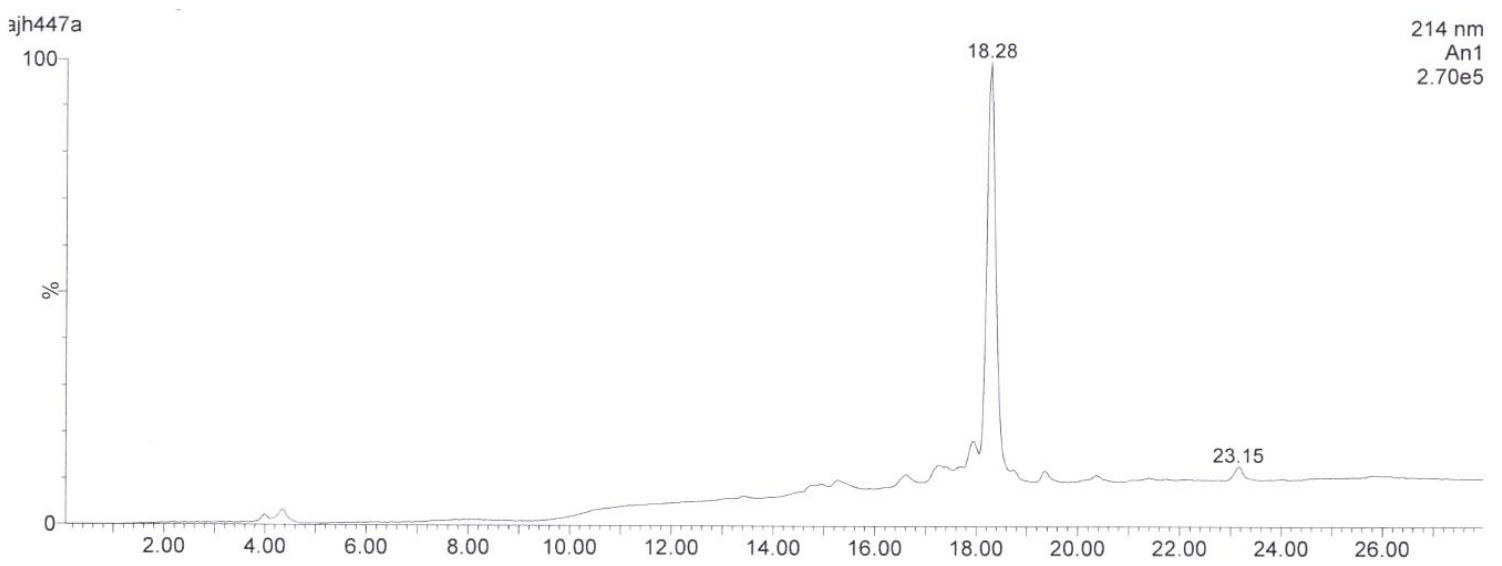

12d

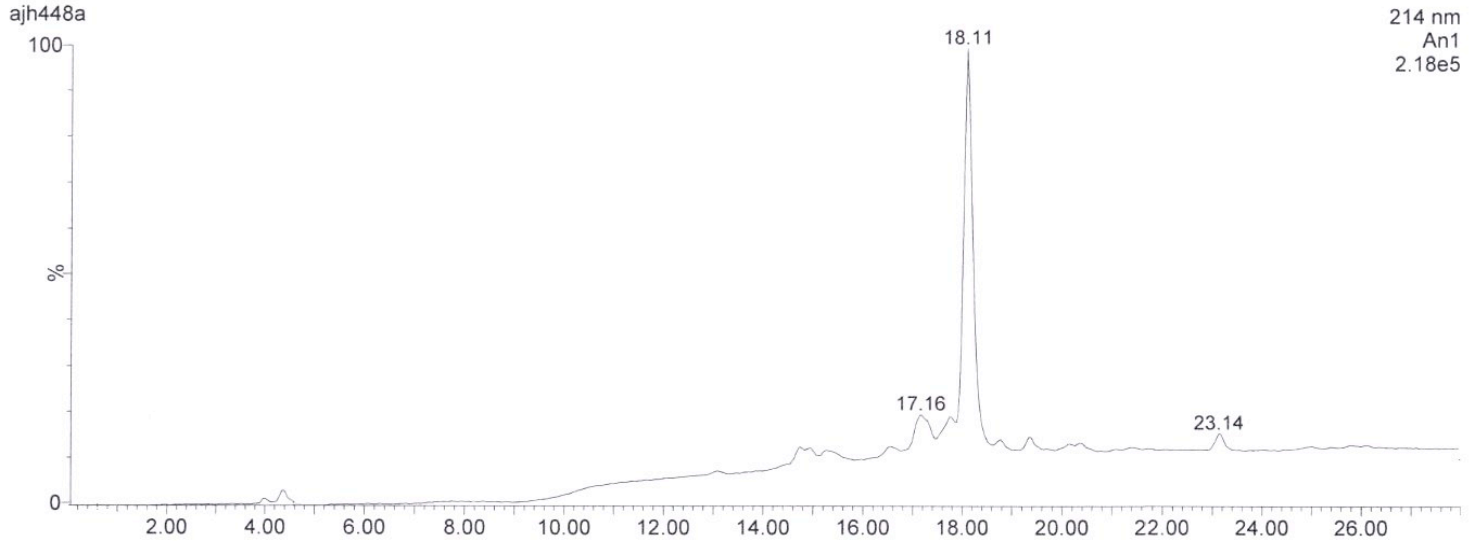

13

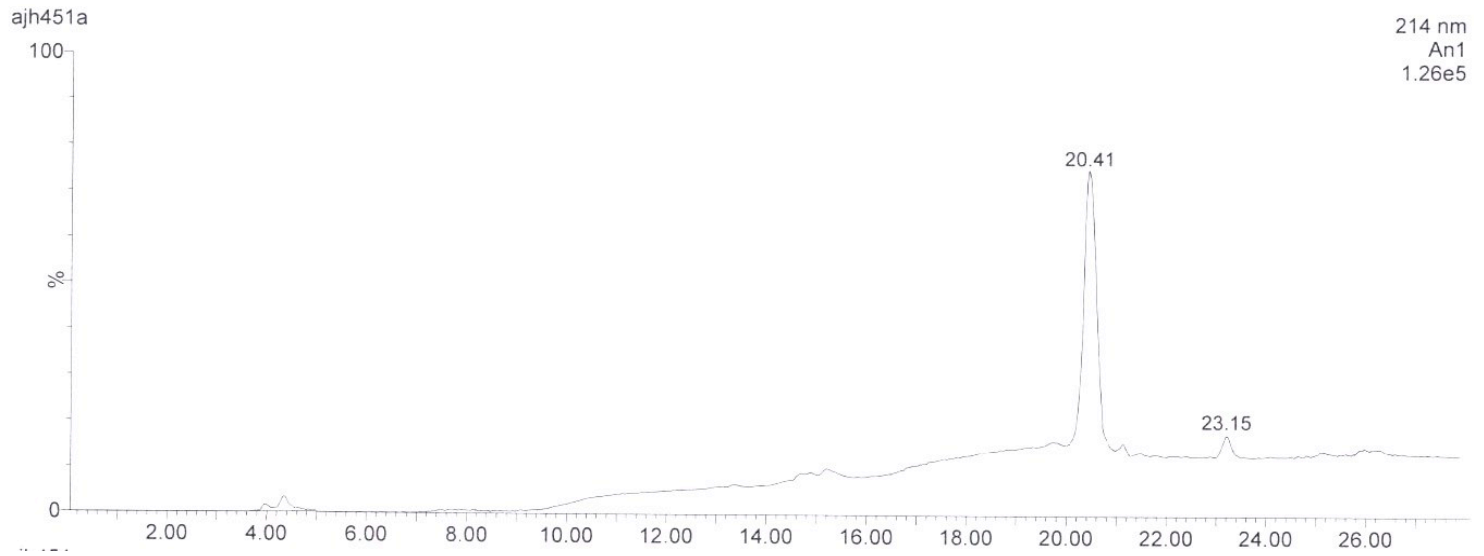




\section{Exact Mass Data}

Compounds 12a-12d were converted to their ethyl esters (on a $1 \mathrm{mg}$ scale) by treatment with $3 \%$ $\mathrm{HCl}-\mathrm{EtOH}(0.5 \mathrm{ml})$ at $10{ }^{\circ} \mathrm{C}$ for $1 \mathrm{~h}$. The solvent was removed under dry $\mathrm{N}_{2}$. The masses of the corresponding monoethyl esters were as follows.

12a: MS (Thioglycerol): $\mathrm{MH}^{+} 925.40241$ calculated for $\mathrm{C}_{45} \mathrm{H}_{61} \mathrm{~N}_{6} \mathrm{O}_{13} \mathrm{~S}: 925.40173$

12b: MS (Thioglycerol): $\mathrm{MH}^{+} 924.41674$ calculated for $\mathrm{C}_{45} \mathrm{H}_{62} \mathrm{~N}_{7} \mathrm{O}_{12} \mathrm{~S}: 924.41772$

12c: $M S$ (Thioglycerol): $\mathrm{MH}^{+} 1038.48587$ calculated for $\mathrm{C}_{51} \mathrm{H}_{72} \mathrm{~N}_{7} \mathrm{O}_{14} \mathrm{~S}: 1038.48580$

12d: MS (Thioglycerol): $\mathrm{MH}^{+} 1151.57076$ calculated for $\mathrm{C}_{57} \mathrm{H}_{83} \mathrm{~N}_{8} \mathrm{O}_{15} \mathrm{~S}: 1151.56986$ 